\section{Forward to the past: ultrasound might be necessary in some patients with rheumatoid arthritis}

In the January issue of $A R D$, Caporali and Smolen ${ }^{1}$ argue against the use of ultrasound in the management of rheumatoid arthritis (RA), citing the negative findings of the TaSER ${ }^{2}$ and ARCTIC $^{3}$ studies and a lack of standardisation. Even disregarding that the results were not unequivocal, showing a slight, but not statistically significant difference between the ultrasound and control groups after a longer time span, I strongly disagree with the overall conclusion.

There is no doubt that modern disease management has led to excellent outcomes for most patients with RA, with the mutilating disease courses of the prebiological era largely a thing of the past. ${ }^{4}$ However, there are still patients, although few, whose disease defies our expectations and causes considerable structural damage. Notable among these patients are the so-called 'silent progressors', in whom radiological progression occurs despite relative clinical well-being and normalised inflammatory markers. This phenomenon can occur in most joints. Overall, this group is small, yet, clinically relevant. By means of clinical examination, these patients cannot be distinguished from those patients with long-standing RA in remission, in whom a slight synovial swelling persists. Power Doppler sonography (PDS), however, can help to differentiate the two groups.

It is conceivable that intensified treatment of patients who display PDS activity without any joint pain could lead to an improved outcome; on the other hand, intensifying treatment for every patient with swollen, but painless joints, would lead to overtreatment of patients with residual swelling without inflammatory activity. This would have negative effects both concerning costs and adverse events.

Admittedly, I know of no study in which ultrasound-guided therapy has improved outcome in these patients, or even whether any systemic or intra-articular therapy has a relevant effect on synovitis in these joints. On the other hand, this could just as well be due to the lack of designated studies, rather than to a lack of effect in itself. In a large-scale, population-based study, the patient group in question is too small to have a significant impact on overall results, which is a likely explanation for the negative results of the TaSER and ARCTIC trials. ${ }^{23}$ It follows that any study exploring the ideal treatment of 'silently progressing RA' must necessarily be performed on such patients only, with the therapy and control group being phenotypically similar; for example, patients with low clinical and serological activity, but with positive PDS and/ or MRI scans indicating subclinical synovitis. ${ }^{5}$ Due to the diversity of affected joints, a standardised ultrasound protocol is unlikely to be suitable for such a study and a more pragmatic approach seems prudent. Only when such a study has been performed, the efficacy on ultrasound-guided therapy for these patients can be adequately evaluated. ${ }^{6}$

Hence, although Caporali and Smolen are probably right that ultrasound surveillance of RA is not necessary on a general basis, there is a relevant number of patients in whom adequate treatment might be ultrasound dependent. Categorically, dismissing ultrasound in patient management and reserving it for diagnostic and differential diagnostic issues would mean that these patients are denied the treatment they need. Rather, we need to identify these patients using all the tools at our disposal and to design studies that address their situation specifically.

\section{Ottar Gadeholt}

Correspondence to Dr Ottar Gadeholt, Department of Rheumatology, University Clinic Würzburg, Würzburg 97080, Germany; gadeholt_o@ukw.de

Funding This research received no specific grant from any funding agency in the public, commercial or not-for-profit sectors.

Competing interests None declared.

Patient consent Not required.

Provenance and peer review Not commissioned; internally peer reviewed.

(c) Article author(s) (or their employer(s) unless otherwise stated in the text of the article) 2019. All rights reserved. No commercial use is permitted unless otherwise expressly granted.

Check for updates

To cite Gadeholt O. Ann Rheum Dis 2019;78:e56.

Received 20 February 2018

Accepted 21 February 2018

Published Online First 6 March 2018

Ann Rheum Dis 2019;78:e56. doi:10.1136/annrheumdis-2018-213278

\section{REFERENCES}

1 Caporali R, Smolen JS. Back to the future: forget ultrasound and focus on clinical assessment in rheumatoid arthritis management. Ann Rheum Dis 2018;77:18-20.

2 Dale J, Stirling A, Zhang R, et al. Targeting ultrasound remission in early rheumatoid arthritis: the results of the TaSER study, a randomised clinical trial. Ann Rheum Dis 2016;75:1043-50.

3 Haavardsholm EA, Aga AB, Olsen IC, et al. Ultrasound in management of rheumatoid arthritis: ARCTIC randomised controlled strategy trial. BMJ 2016;354:14205.

4 The Lancet. A platinum age for rheumatology. Lancet 2017;389:2263.

5 Sewerin $\mathrm{P}$, Vordenbaeumen $\mathrm{S}$, Hoyer $A$, et al. Silent progression in patients with rheumatoid arthritis: is DAS28 remission an insufficient goal in RA? Results from the German Remission-plus cohort. BMC Musculoskelet Disord 2017;18:163.

6 Nguyen H, Ruyssen-Witrand A, Gandjbakhch F, et al. Prevalence of ultrasound-detected residual synovitis and risk of relapse and structural progression in rheumatoid arthritis patients in clinical remission: a systematic review and meta-analysis. Rheumatology 2014;53:2110-8. 